\title{
Imaging Spectrum of Soft Tissue Lesions of Extremities
}

\author{
Shyam S ${ }^{1}$, Kumar Ashok Charan², Parthasarathy K R ${ }^{3}$, Daneshwari Umadi ${ }^{4}$, Mehathab M Bava ${ }^{5}$ \\ ${ }^{1}$ Assistant professor, Department of Radio-Diagnosis, SSIMS \& RC, Davangere, ${ }^{2}$ Assistant professor, Department of Radio- \\ Diagnosis, Dr B R Ambedkar Medical College, Bengaluru, ${ }^{3}$ Professor and HOD, Department of Radio-Diagnosis, SSIMS \& RC, \\ Davangere, ${ }^{4}$ Resident, Department of Radio-Diagnosis, SSIMS \& RC, Davangere, ${ }^{5}$ Resident, Department of Radio-Diagnosis, \\ SSIMS \& RC, Davangere
}

Corresponding author: Dr. Kumar Ashok Charan, Assistant Professor, Department of Radiodiagnosis, Dr B R Ambedkar Medical college, Bengaluru, Karnataka, India

DOI: http://dx.doi.org/10.21276/ijcmsr.2019.4.4.33

How to cite this article: Shyam S, Kumar Ashok Charan, Parthasarathy K R, Daneshwari Umadi, Mehathab M Bava. Imaging spectrum of soft tissue lesions of extremities. International Journal of Contemporary Medicine Surgery and Radiology. 2019;4(4):D139-D145.

\section{A B S T R A C T}

Introduction:Soft-tissue lesions are frequently encountered by radiologists in everyday clinical practice. Clinical details and imaging characteristics on various imaging modalities has been methodically assessed toestablish the diagnosis for lesions that have typical clinical and imaging features and narrow the differential diagnosis. The aim of this article was to aid radiologist in characterizing the imaging features soft tissue lesion

Material and methods: In this prospectivestudyultrasound and MRI with correlative radiography and computed tomography was carried out on 40 patients who were referred to radiology with suitable clinical diagnosis. Final diagnosis was done with the histo-pathological findings.

Results: Among 40 patients in the present study, $68 \%$ cases were malignant, and $32 \%$ cases were benign in which the common age group was between 31-40 years in both malignant and benign patients.

Conclusion:Soft-tissue lesions are routinely faced by radiologists in daily clinical practice. On imaging depiction of these softtissue lesions remains problematic. By detailedly using clinical history and various imaging features help in determine the diagnosis or narrow the differential diagnosis. From the present study, we conclude that Magnetic Resonance Imaging (MRI) is a well-established imaging tool for the characterization of soft-tissue tumors. In this article a review of latest updated classification of soft tissue tumour has been discussed.

Keywords: Magnetic Resonance Imaging, Ultrasound, Soft tissue lesions

\section{INTRODUCTION}

Soft tissue lesions range from non-neoplastic conditions to benign and malignant tumors. Soft tissue tumors are classified histologically on the basis of the adult tissue they resemble. ${ }^{1,2}$ Lesion emerging from the fat does not nominate of lipo-sarcoma, but rather that it is a malignant mesenchymal tumor that has differentiated into tissue that microscopically similar to normal adult fat. ${ }^{3}$ Since deficient in microscopic features needed for accurate diagnosis, many sarcomas are poorly distinguished. In such cases, immune histo-chemical stains have aided pathologists in identifying their pattern of differentiation, allowing accurate classification. Despite the pathologist's best efforts, however, approximately 5-15\% of soft-tissue sarcomas cannot be furtherclassified. ${ }^{3-6}$ Hardly $1 \%$ of all tumours make up the soft tissue tumours. The annual incidence of soft tissue tumors is approximately 300 per 100,000 people. Soft tissue sarcomas arise most commonly in the extremities, chest wall and retroperitoneum and are more common in older people and males, although age and gender vary for the various histological types.Soft-tissue lesions are frequently encountered by radiologists in everyday clinical practice. Clinical details and imaging characteristics on various imaging modalities has been methodically assessed toestablish the diagnosis for lesions that have typical clinical and imaging features and narrow the differential diagnosis. Current research aimed to study MRI characteristics of different soft tissue tumors and to assess the local tumor staging of soft tissue tumor, to aid surgeons by extensions of the lesion with surrounding neurovascular bundles and bone and to identify purely benign soft tissue lesion so that unwanted biopsy and surgery will be avoided.

\section{MATERIAL AND METHODS}

In this prospective study ultrasound and MRI with correlative radiography and computed tomography was carried out on 4 0patients who were referred to radiology at S.S. Institute of medical sciences Davangere and clinical images and inputs were from Dr B R Ambedkar Medical College, Bengaluru with suitable clinical diagnosis. Final diagnosis was done with the histo-pathological findings. The study period was 2 years (May 2017 to May 2019). This study was based on prospective analysis of 40 patients with soft tissue lesions. A patient who fulfils the inclusion and exclusion criteria was selected for the study. 
Inclusion criteria

1. Patients with soft tissue lesion

2. Either sex

\section{Exclusion criteria}

1. Pregnant women;

2. Patient having history of claustrophobia.

3. Patient having history of metallic implants insertion, cardiac pacemakers and metallic foreign body insitu.

\section{Procedure methodology}

After necessary required consent were obtained and subjected to, ultrasound and MR imaging with correlative radiography or computed tomography whenever requiredwas performed at our institution. Magnetic resonance imaging were carried out on a 1.5-T MRI system (GE Signa 1.5T).

\section{RESULT}

In the present study, $68 \%$ cases were malignant, and $32 \%$ cases were benign in which the common age group was between 31-40 years in both malignant and benign patients. Parameters are most consistently associated with malignancy with higher sensitivity, specificity and PPV are size $>8 \mathrm{~cm}$, $\mathrm{T} 2 \mathrm{w}$ heterogeneous hyper intensity, heterogeneous contrast enhancement, osseous and neurovascular involvement, peri-tumoraledema, Intralesional necrosis and ill-defined

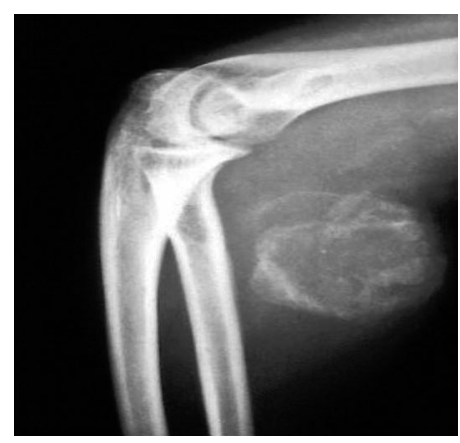

Figure-1: margins. So MRI can be considered as modality of choice for the evaluation of soft tissue tumors which his highly sensitive in detection. It must be stress that MR imaging

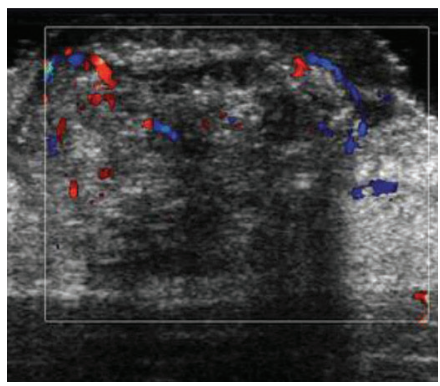

Figure-2:

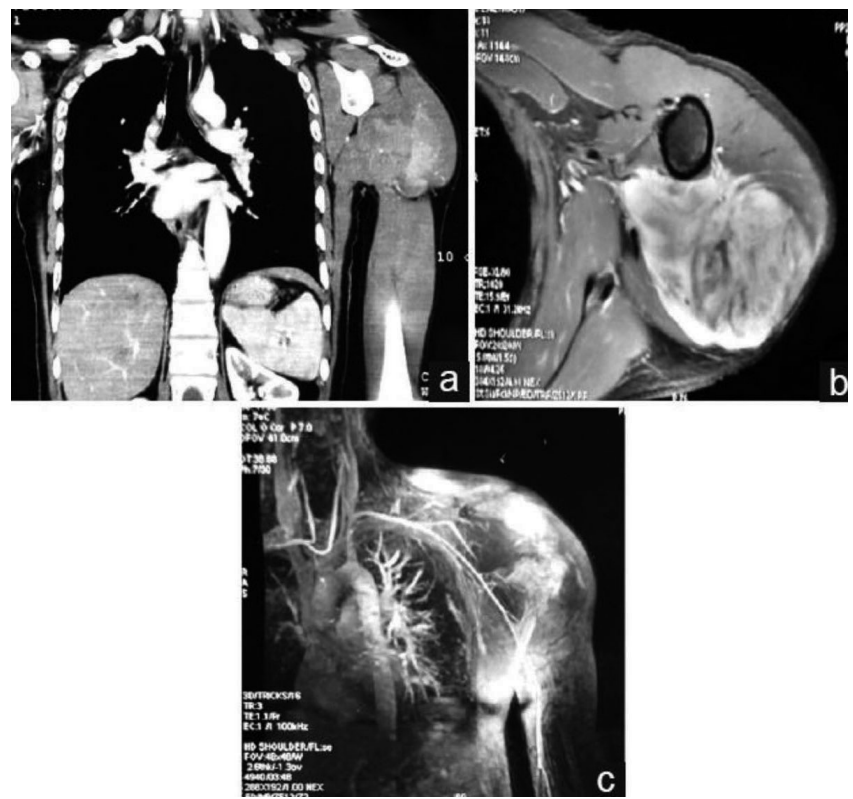

Figure-3: Courtesy: Azam M, Khurana R, Gupta A. An unusual case of pleomorphic rhabdomyosarcoma of shoulder in an adult patient. Clinical Cancer Investigation Journal. ${ }^{5}$

\begin{tabular}{|c|c|}
\hline Tumor category & Major changes \\
\hline Adipocytic & Mixed-type liposarcoma removed \\
\hline Fibroblastic & DFSP and giant cell fibroblastoma included for the first time \\
\hline \multirow[t]{2}{*}{ Myofibroblastic } & "Hemangiopericytoma" removed as a synonym for SFT \\
\hline & Recognition of nodular fasciitis and variants as trueneoplasms \\
\hline So-called fibrohistiocytic & "Malignant fibrous histiocytoma" removed \\
\hline Smooth muscle & Angioleiomyoma reclassified as pericytic tumor \\
\hline \multirow[t]{2}{*}{ Pericytic } & Angioleiomyoma reclassified as a pericytic tumor \\
\hline & Myofibroma now classified as pericytic tumor \\
\hline \multirow[t]{2}{*}{ Vascular } & Pseudomyogenic (epithelioid sarcoma-like) \\
\hline & Hemangioendothelioma added as a new entity \\
\hline Gastrointestinal stromal & GIST included in the volume on STT for the first time \\
\hline \multirow[t]{2}{*}{ Nerve sheath } & Peripheral nerve sheath tumors included volume on STT for the firsttime \\
\hline & New hybrid benign nerve sheath tumors included (schwannoma/ perineurioma) \\
\hline \multirow[t]{4}{*}{ Tumors of uncertain differentiation } & New tumors: acralfibromyxoma, hemosideroticfibrolipomatous tumor differentiation \\
\hline & Phosphaturicmesenchymal tumor \\
\hline & Atypical fibroxanthoma now included \\
\hline & PNET removed as synonym for Ewing"s sarcoma \\
\hline Undifferentiated/other category & Includes tumors that cannot be classified into any unclassified sarcoma \\
\hline
\end{tabular}




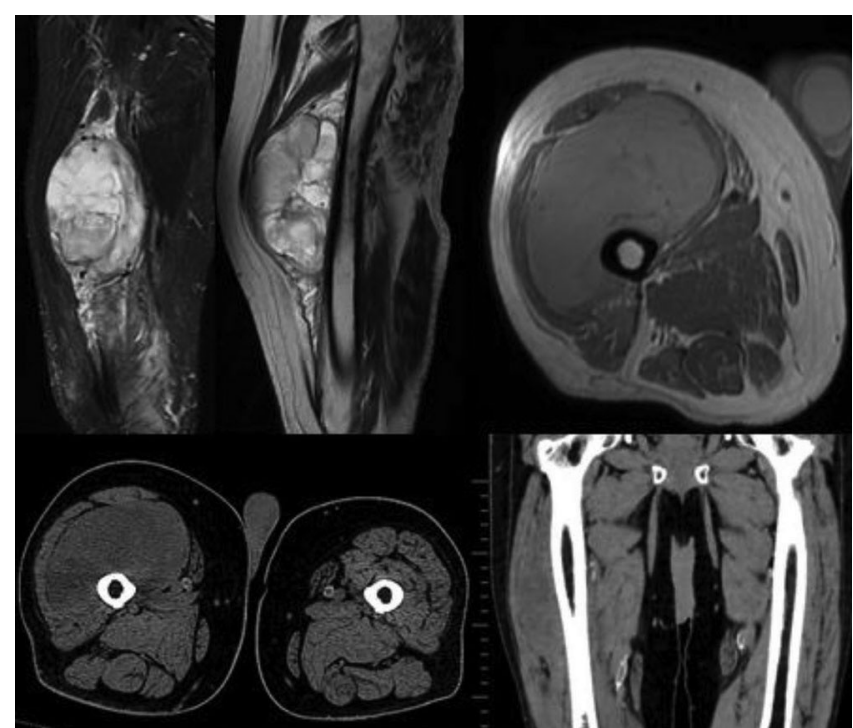

Figure-4: 65year old male with history of right thigh swelling, $\mathrm{CT}$ and MR revealed Malignant fibrous histiocytoma ${ }^{5}$
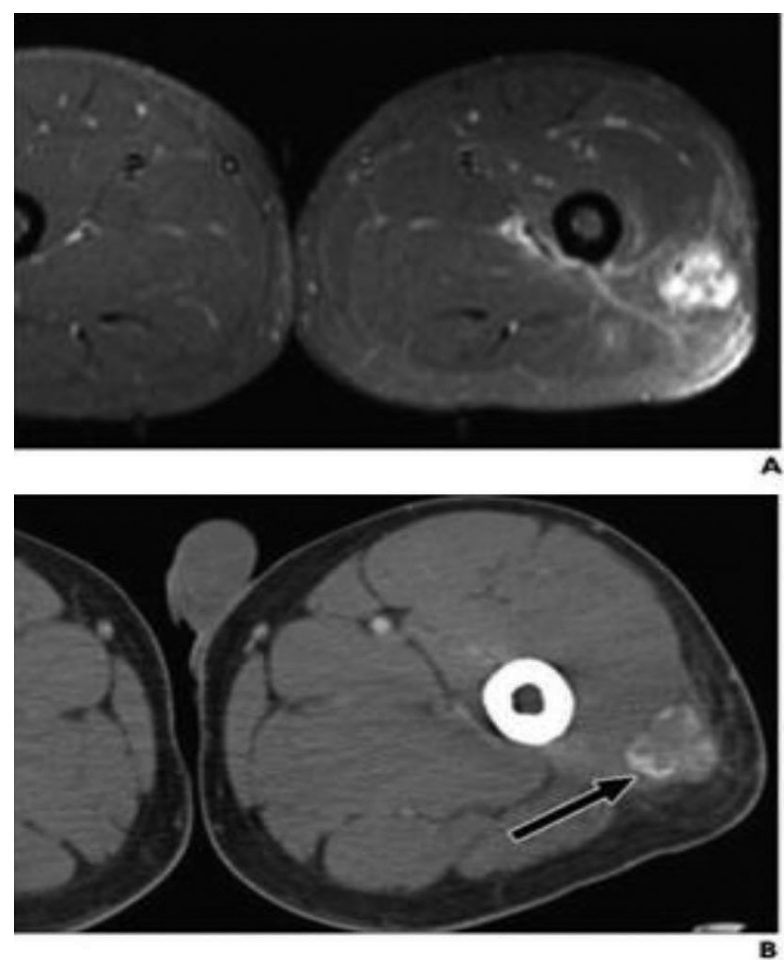

Figure-5: 21 year old man presenting with several week long history of painful lesion with progressive increase in size with no history of trauma and no significant radiography finding. (A) MRI shows non specific soft tissue mass in posterolateral thigh with diffuse enhancement and surrounding edema as seen on fat saturated axial T1 weighted image obtained after administration of gadolinium contrast agent. (B) Follow up CT suggests final diagnosis of heterotopic ossification (myositis ossificans) by revealing characteristic peripheral zone of ossification.

cannot completely distinguish benign and malignant lesions and when radiologic evaluation is nonspecific. MR imaging may provide a specific diagnosis in a limited number of tumours. Differential diagnosis is established whenever specific diagnosis is not possible.

\section{DISCUSSION}

Soft tissue originate mainly from mesenchyme and by accord composed of fibrous tissue, fat, skeletal muscle, vascular structures and peripheral nervous system. ${ }^{1}$ Neoplastic growth of soft tissue arises from the non-epithelial extra skeletal connective tissue of the body like tendons, blood vessels and musclesare usually mesodermal in origin.Various imaging features has been described.

\section{Radiograph}

Radiography is the evaluation of choice despite imaging advances in our era and in all suspected soft-tissue mass must begin with a radiograph. Even though not much details will depicted with radiography and most often it will beunrewarding. May be helpful in clinically palpable lesion caused by an underlying skeletal deformity, which may disguise as a soft-tissue mass. Soft-tissue calcifications can be portrayed well with radiograph. For example, they may reveal juxtaarticularosteocartilaginous masses of synovial chondromatosis, peripherally more mature ossification of myositis ossificans, phleboliths within a hemangioma or characteristic bone changes of other processes with associated soft- tissue involvement.Lateral radiograph of a 30 year male presented with swelling around elbow showing soft tissue sarcoma has been depicted in figure 1

\section{Ultrasound}

Ultrasonography is the routine investigation sorted after radiograph in a clinical suspected case of soft tissue tumors of the extremities Accuracy of ultrasound is high in the assessment of soft tissue. Accuracy also depends on sound knowledge of the radiologist. Due to advance imaging methods clinician will request for the same, hence whenever a suspected case of soft tissuetumour has been requested for MRI or CT, a correlative ultrasound will help in further clarify the ultrasound features. Superficial probe is usually used for the examination. 15year old boy with forearm mass, colour doppler shows moderate degree of intrinsic vascularity. Mass was initially diagnoses as hemangioma is depicted in figure 2. Final histologic diagnosis was pilomatricoma.

\section{Omputarised tomography}

Computed tomography has a major role characterization of soft-tissue lesion. Bone involvementof soft-tissue tumors can be best evaluated. With $3 \mathrm{D}$ reconstructions, further adds to the evaluation. Correlative CT plays complimentary role in diagnosis of these masses, and delineate what information may be gained for treatment planning. Figure 3: Coronal CTshows heterogenous mass lesion in left shoulder region (b) T2-weighted axial section showing iso- to hyper-intense soft tissue mass lesion. (c) Magnetic resonance spectroscopy image showing multiple feeding channels supplying the neoplastic lesion arising from axillary and subclavian arteries and their branches. ${ }^{5}$

Four discriminating features are:

1. Mineralization pattern

2. Density

3. Pattern of bone involvement

4. Lesion vascularity. 


\section{Anatomy and MRI appearance of soft tissue}

Soft tissue tumor is a neoplastic growth that usually are mesodermal in origin, that is non-epithelial extra skeletal connective, soft tissues of the body, such as the muscles, tendons and blood vessels which. Neoplastic growth of soft tissue arises from the non-epithelial extra skeletal connective tissue of the body like tendons, blood vessels and muscles are usually mesodermal in origin. Mainly elastin and collagen are the constituents of soft tissue. In addition non-cellular, fibrous components of a cell also make up part of this tissue. Elasticity and hydrated state of the cells are sorting of these cells.

\section{Classification and features of soft tissue tumors}

Soft-tissue sarcomas are a rare heterogeneous group of malignancies that account for almost $20 \%$ of paediatric and $1 \%$ of adult malignancies, approximately $20-40 \%$ of which occur in the torso. ${ }^{3}$ The Surveillance, Epidemiology, and End Results (SEER) database estimates that there were 13,000 new cases of soft-tissue sarcomas in 2012 in the United States. ${ }^{6}$ Although commonly perceived to be exceedingly rare, soft-tissue sarcomas are comparable in annual incidence to other cancers such as esophageal (17,500 new cases), gastric (21,000 new cases), and cervical (12,000 new cases) cancers. 34 The reported incidence of soft-tissue sarcomas is also likely underestimated. For example, in a prospective population-based study performed in France between March 2005 and February 2007, Ducimetièreet al. ${ }^{7}$ performed a centralized review of molecular analyses of all suspected cases across the region and concluded that the observed incidence of sarcomas was higher than expected. The study found a crude incidence rate and world age-standardized incidence rate of 6.4 and 4.8 cases per 100,000, respectively, which are higher than the 1-3 cases per 100,000 reported in most prior studies in the United States and Europe ${ }^{3-4}$, and an overall incidence of $1.3 \%$ of all new cancers in the region. Gastrointestinal stromal tumors (GISTs)(18\%), unclassified sarcomas (16\%), and liposarcomas (15\%) were the most common sarcomas in the Ducimetière et al. ${ }^{7}$ study, and $40 \%$ of the soft-tissue sarcomas occurred in the torso.

\section{Revised WHO Classification}

Soft tissue tumors (STT) represent a complex group of lesions that may show a broad range of differentiation. The WHO (World Health Organization) classification was established and up-to-dated in 2013 for the purpose of uniformity. ${ }^{5}$ The WHO system helps to unify the lexicon for the performance of clinical trials and toserveasa guide for the multidisciplinary working group of specialists such as radiologists, pathologist and orthopediconcologists, to improve the patient "management and outcome. The nomenclature has been adapted by the American Joint Cancer Commission (AJCC) for sarcoma staging and by the College of American Pathologists Cancer protocols for soft tissue sarcomas. The 2013 classification is the 4th edition and replaces the previous edition in $2002^{7}$ The major modifications from the previous edition are the addition of three new chapters: gastrointestinal stromal tumors (GIST), nerve sheath tumors, and undifferentiated/unclassified sarcomas.
The WHO classification incorporates detailed clinical, histological, and genetic data. There are also imaging features in the new edition of soft tissue tumor classification. The classification of soft tissue tumors of the WHO includes the following groups:

1. Adipocytictumors

2. Fibroblastic/myofibroblastic tumors

3. So-called fibrohistiocytic tumors

4. Smooth muscle tumors,

5. Pericytic (perivascular) tumors,

6. Skeletal muscle tumors,

7. Vasculartumors,

8. Gastrointestinal stromal tumors,

9. Nervesheathtumors,

10. Chondroosseoustumors,

11. Tumors of uncertain differentiation

12. Undifferentiated/unclassified sarcomas.

Generally, the WHO classification divides soft-tissue tumors in four categories according to biological potential. ${ }^{7}$

\section{Benign Tumors}

- Do not recur or metastasize after resection

- Recurrence is non-destructive if it occurs

- Example: Lipoma.

\section{Intermediate Locally Aggressive Tumors}

- Locally infiltrative and destructive

- Often recur after resection but do not metastasize

- Example: Desmoid tumor, well-differentiated liposarcoma.

\section{Intermediate Rarely Metastasizing Tumors}

- Locally aggressive and can recur

- These sarcomas metastasize in less than $2 \%$ of cases

- Example: dermato fibrosarcoma protuberans [DFSP]).

\section{Malignant Tumors}

- Most common type of soft-tissue tumor.

- Recur with a high risk of metastasis

- Example: GIST, myxoidliposarcoma.

The new classification has incorporated more detailed cytogenetic and molecular data in accordance with the rapidly increasing knowledge of genetics of tumors. Softtissue sarcomas are divided into several categories $5,6,7,8$

Note: WHO-World Health Organization, DFSP-dermato fibrosarcoma protuberance, SFT-solitary fibrous tumor, GIST-gastrointestinal stromal tumor, STT-soft tissue tumor, PNET-primitive neuroectodermal tumor

\section{Adipocytic Tumors}

- These group of tumors constitute the largest incidence of mesenchymal /soft tissue tumors.

- Examples are lipomas, angiolipomas, and liposarcomas, adipocytic tumors.

- Atypical lipoma and liposarcoma are considered similar by WHO classification and considered locally aggressive with no potential for metastasis. ${ }^{6,8}$

- The terms "mixed-type liposarcoma" and myxolipoma have beendeleted.

- Diffuse lipoblastoma is now the better term for lipoblastomatosis in pediatrics.

- Alterations been done in the clarity of the term 'differentiated liposarcoma' and is now been subdivided 
into four groups (see Table 1).

- Chondroid lipoma may be similar tomyxoidliposarcoma. Chondroid lipoma mostly has calcifications, which are best demonstrated by radiographs or computed tomography.

\section{Fibroblastic/Myofibroblastic Tumors}

- Constitutelarge group of mesenchymal tumors, mostly contain fibroblastic as well as myofibroblastic elements. ${ }^{6}$

- Nodular fasciitis, proliferative fasciitis, and proliferative myositis are identified as neoplastic in the current WHO classification,previously they were taught to be reactive lesions.

- Other changes were the inclusion of the closely related giant cell fibroblastoma and dermatofibrosarcomaprotuberans (DFSP), formerly included in volume on skin lesions. DFSP is categorized as a rarely metastasizing (intermediate) tumor, although it should be noted that metastatic potential is gained only when a component of a fibrosarcomatous change is present. ${ }^{10}$

- There are $50 \%$ chances of recurrence in case of giant cell fibroblastoma, hence it has been grouped in the locally aggressive (intermediate) category. ${ }^{10}$ But giant cell fibroblastoma;s do not metastasize.

- Hemangiopericytoma has beendeleted from the classification

- Lipomatoushemangiopericytoma and giant cell angiofibromahave also been included as soft tissue tumours.

- The term "atypical myxoinflammatory fibroblastic tumor" was introduced for as synonym for "myxoinflammatory fibroblastic sarcoma." This new term better reflects the extremely low potential for metastasis for this tumor type. ${ }^{11}$

\section{So-Called Fibrohistiocytic Tumors}

- Malignant fibrous histiocytoma (MFH) has been removed from the current WHO classification.

- $\mathrm{MFH}$ and its subtypes have been reclassified in the new separate group of unclassified/undifferentiated sarcomas (group 12). ${ }^{6}$ An example for MFH has been depicted on figure 4.

- Malignant tumours has not been included in group 3. It includes the common tenosynovial giant cell tumors, the uncommon giant cell tumor of soft tissues, and the plexiformfibrohistiocytic tumor.

\section{Smooth Muscle Tumors}

- No important changes in this category.

- Smooth muscle tumors arising at non-cutaneous, nonuterine locations have been the focus of aappreciable conceptual shift in the past.

- Angioleiomyoma (vascular leiomyoma) was redistributed to the category of pericytic (perivascular) tumors, this was the only changes.

\section{Pericytic (Perivascular)Tumors}

- This category has been first included in 2002 edition, during whichhemangiopericytoma was deleted from this group. But was listed as synonym for solitary fibrous tumor (group 2).
- In this group, Glomustumor are infrequent but most common tumor categorized on imaging. They are made up of cells like the modified smooth muscle cells of the normal glomus body.

- Angioleiomyoma (vascular leiomyoma) has been added on to this group. ${ }^{11}$

\section{Skeletal Muscle Tumours}

- Two entities have been added: rhabdomyoma and rhabdomyosarcoma.

- Rhabdomyoma is subdivided into cardiac and extracardiac types and pathologically as adult, fetal (immature skeletal muscle fibers), and genital (female or male genital tract).

- Extracardiac adult rhabdomyoma is rarely reported on imaging.

- Spindle cell/sclerosingrhabdomyosarcoma is recognized as a separate tumour different from embryonalrhabdomyosarcoma.

\section{Vascular Tumours}

- Benign vascular tumours are very common and most frequently occur in the skin.

- It is often difficult to determine whether they represent malformations, true neoplasms, or reactive processes. ${ }^{13}$

- Hemangiomas are classified histologically as synovial, venous, arteriovenous, mixed malformations, and intramuscular.

- "Pseudomyogenic (epithelioid sarcoma-like) hemangioendothelioma" has been added as a rarely metastasizing neoplasm.

- These tumours are mainly seen in $2^{\text {nd }}$ to $3^{\text {rd }}$ decade of age with presentation of multi-site nodules in different planes of a limb and involve the cutaneous as well as deep soft tissues and the bone.

- This tumour tends to be highly FDG-avid, and therefore PET-CT is useful for the detection of deep lesions.

\section{Chondro-Osseous Tumours}

- There are no changes on the chondro-osseous soft tissue tumours.

- Myositis ossificans was regarded as a (myo)fibroblastic lesion in the 2002 version, and extra skeletal myxoidchondrosarcoma (EMC) was also classified in the tumours of uncertain differentiation, since it shows little evidence of cartilaginous differentiation, despite the name.

- Mesenchymalchondrosarcoma of soft tissues occurs less frequently than the EMC, but nearly half of them esenchymalchondrosarcomasare extraskeletalin location. ${ }^{12}$

- An example for myositis ossificans has been depicted in figure 5 .

- Extraskeletal osteosarcoma, soft tissue osteosarcoma, shows similar histologic features of bone osteosarcoma without systematic geneticdifferences. ${ }^{10}$

\section{Gastrointestinal Stromal Tumours}

- This group has been added, gastrointestinal stromal tumour (GIST), which is the most common primary mesenchymaltumour in the gastrointestinal tract. 
- Histologically GIST are classified as benign, uncertain malignant potential, and malignant.

- Prognostic factors are tumour size, mitotic activity, and anatomical site.

- Almost 50\% arise in the stomach, $30 \%$ in the small intestine, and the rest for the colon, rectum, and esophagus and primary disseminated with unspecified site of origin.

- Isolated cases have been reported in the appendix. As GIST present with abdominal symptoms and are detected by ultrasound or CT scans of the abdomen, these lesions are not referred to as musculoskeletal tumours as such.

\section{Peripheral Nerve Sheath Tumors}

- Nerve sheath tumours were previously included in the 2007 WHO classification of tumours of the central nervous system.

- Although imaging reviews on soft tissue tumours already regarded nerve sheath tumours previously as typical soft tissue tumors, nerve sheath tumours have been included for the first time in the WHO classification of soft tissue tumours since 2013.

- Hybrid nerve sheath tumours, such as schwannoma/ perineurioma ${ }^{14}$ and neurofibroma/ schwannoma, have been included in the group of peripheral nerve sheath tumours.

- The latter might be related to NF-2, NF-1, orschwannomatosis. ${ }^{15}$

\section{Tumours of Uncertain Differentiation}

- Tumors with unknown clear line of cell differentiation are included in this group. It includes a long list of tumors.

- New entities have been included: acralfibromyxoma (digital fibromyxoma), hemosideroticfibrolipomatous tumor, phosphaturicmesenchymal tumor, and atypical fibroxanthoma.

- Among the group of malignant lesions, primitive neuroectodermal tumor (PNET) has been dropped as a synonym for Ewing's sarcoma in order to minimize confusion with similarity named lesions in the CNS and female genital tract.

- Extraskeletalmyxoidchondrosarcoma is included in this category as there is no convincing evidence of cartilaginous differentiation. ${ }^{16}$

- Deep ("aggressive") angiomyxoma is an uncommon slowly growing neoplasm with a predilection for pelvic and perineal regions and tendency to local recurrence and characteristic features on MR.

- Ossifying fibromyxoid tumor of the soft tissue is a wellcircumscribed lobulated hard tumor covered by a thick fibrous pseudocapsule.

- It was surprising that the term "synovial sarcoma" remained unchanged in the current updated classification.

- As the lesion is not derived from true synovial cells and may involve virtually any body part, the term "synovial sarcoma" is indeed a misnomer.

- Therefore, future revisions of the WHO classification on STT should consider to abandon the confusing term "synovial sarcoma."

\section{Undifferentiated/Unclassified Sarcoma}

- This new category of tumors, introduced for the first time in the 2013 classification, recognizes the fact that a small, but significant, subset of sarcomas cannot be classified into any presently defined categories. ${ }^{17}$

- This group of tumors was previously included in the fibrohistiocytic group (group 3), namely, "malignantfibrous histiocytoma."

- This group of tumors might have spindle cell, pleomorphic, round cell, or epithelioid morphology.

- A subset of radiation-associated sarcomas falls into this category.

- These lesions show no definable line of differentiation using currently available technologies.

- Dedifferentiated types of specific sarcomas are not included in this category.

- Undifferentiated/unclassified sarcoma accounts for up to $20 \%$ of all sarcomas and about a quarter of these are radiation-associated tumors.

- It is likely that this group of lesions will be subject to future reclassification along with ongoing progress in molecular genetics.

\section{CONCLUSION}

Radiologists come across soft-tissue lesions routinely in everyday practice, despite advances in imaging distinguishing of these soft-tissuelesions remains problematic.Clinicaldetails and imaging characteristics on various imagingmodalities has been methodically assessed to establish the

diagnosis for lesions that have typical clinical and imaging features and narrow the differential diagnosis. From the present study, we conclude that Magnetic Resonance Imaging (MRI) is a well-established imaging tool for the detection and local staging of soft-tissue tumours. MR imaging exhibited different advantages like determining the origin of these lesion in defining their extent and relation to adjacent structures, assessing operability by identifying osseous, neurovascular bundles and joint space involvement by soft tissue tumors. In addition computed tomography or radiograph can be correlatively performed wherever required to further narrow down the diagnosis. As a result, MR images can be particularly useful for characterizing lesions that do not require imaging follow-up or biopsy by pattern recognition. Also a review on latest updated classification of soft tissue tumour has been portrayed.

\section{REFERENCES}

1. Wu JM, Montgomery E. Classification and pathology. SurgClin North Am. 2008;88(1):483-520.

2. Murphey MD. World Health Organization classification of bone and soft tissue tumors: modifications and implications for radiologists. Semin Musculoskelet Radiol. 2007;11(3):201-214.

3. Chen CK, Yeh LR, Pan HB, et al. Intra-articular gouty tophi of the knee: CT and MR imaging in 12 patients. Skeletal Radiol. 1999;28(5):75-80.

4. Steinbach LS, Johnston JO, Tepper EF, Honda GD, Martel W. Tumoralcalcinosis: radiologic-pathologic 
correlation. Skeletal Radiol. 1995;24(4):573-578.

5. Azam M, Khurana R, Gupta A. An unusual case of pleomorphic rhabdomyosarcoma of shoulder in an adult patient. Clinical Cancer Investigation Journal. 2016;5(3):274.

6. Baheti AD, O'Malley RB, Kim S, Keraliya AR, Tirumani SH, Ramaiya NH, Wang CL. Soft-tissue sarcomas: an update for radiologists based on the revised 2013 World Health Organization classification. American Journal of Roentgenology. 2016;206(5):924-32.

7. Mastrangelo G, Coindre JM,Ducimetière F, Dei Tos AP, Fadda E, Blay JY, Buja A, Fedeli U, Cegolon L, Frasson $\mathrm{A}$, Ranchère-Vince $\mathrm{D}$. Incidence of soft tissue sarcoma and beyond: a population-based prospective study in 3 European regions. Cancer. 2012;118(21):5339-48

8. Hayes CW, Conway WF. Calcium hydroxyapatite deposition disease. RadioGraphics. 1990;10(4):10311048.

9. Kraemer EJ, El-Khoury GY. Atypical calcific tendinitis with cortical erosions. Skeletal Radiol. 2000;29(6):690 696.

10. ManasterBJ, May DA, Disler DG. Miscellaneous tumors and tumorlike lesions. In: Thrall J, editor. Musculoskeletal imaging: the requisites. 3. St Louis MO: Mosby; 2006. pp. 523-526.

11. Vanhoenacker FM, Parizel PM, Gielen JL. Imaging of soft tissue tumors. Springer Science \& Business Media; 2006 Apr 26.

12. O'Sullivan PJ, Harris AC, Munk PL. Radiological features of synovial cell sarcoma. $\mathrm{Br} \mathrm{J}$ Radiol. 2008;81(5):346-356.

13. Munk PL, Lee MJ, Janzen DL, et al. Lipoma and liposarcoma: evaluation using CT and MR imaging. AJR. 1997;169(1):589-594.

14. Kransdorf MJ, Bancroft LW, Peterson JJ, Murphey MD, Foster WC, Temple HT. Imaging of fatty tumors: distinction of lipoma and well-differentiated liposarcoma. Radiology. 2002;224(5):99-104.

15. Reiseter T, Nordshus T, Borthne A, Roald B, Naess P, Schistad O. Lipoblastoma: MRI appearances of a rare paediatric soft tissue tumour. PediatrRadiol. 1999;29(4):542-545.

16. Dow N, Giblen G, Sobin LH, Miettinen M. Gastrointestinal stromal tumors: differential diagnosis. InSeminars in diagnostic pathology WB Saunders. 2006;23(2):111-119.

17. Fletcher CD. Undifferentiated sarcomas: what to do? And does it matter? A surgical pathology perspective. Ultrastructural pathology. 2008;32(2):31-6.

Source of Support: Nil; Conflict of Interest: None

Submitted: 06-09-2019; Accepted: 01-10-2019; Published online: 19-12-2019 